\title{
Évaluation de la pratique de la césarienne selon la classification de Robson dans un district sanitaire de Ouagadougou, Burkina Faso
}

\author{
Evaluation of the Practice of Caesarean Section According to Robson's Classification in a Health \\ District of Ouagadougou, Burkina Faso
}

\author{
H. Zamané $\cdot$ S. Kiemtoré $\cdot$ P.D. Kain - A. Zongo · B.M.G. Hounkanri $\cdot$ Y.A. Sawadogo $\cdot$ A. Ouattara \\ I. Ouédraogo $\cdot$ BE Savadogo-Komboigo $\cdot$ C.M.R. Ouédraogo
}

Reçu le 21 janvier 2018; accepté le 3 décembre 2018

(C) Société de pathologie exotique et Lavoisier SAS 2019

\begin{abstract}
Résumé Le contrôle du taux de césariennes est, de nos jours, une préoccupation importante pour le monde obstétrical, la priorité étant de tout mettre en œuvre pour pratiquer une césarienne chez toutes les femmes qui en ont besoin plutôt que d'atteindre un taux spécifique. La présente étude a pour objectif d'appliquer la classification de Robson à l'évaluation de la pratique de la césarienne à la maternité de l'hôpital de district de Bogodogo. Il s'agit d'une étude transversale descriptive sur une période de trois ans, du $1^{\mathrm{er}}$ janvier 2013 au 31 décembre 2015. La base informatique des dossiers de césarienne, les dossiers d'accouchement, les registres du bloc opératoire, de la salle d'accouchement et les rapports mensuels d'activités étaient les sources d'information utilisées. La fréquence hospitalière globale de césarienne était de $33,3 \%$. Le taux de césarienne attendu durant la même période selon le C-Model était de 9,7\%. Les patientes des groupes 5 (avec un utérus cicatriciel) et 6 (nullipares avec
\end{abstract}

H. Zamané $(\bowtie) \cdot$ S. Kiemtoré · P.D. Kain · B.M.G. Hounkanri Y.A. Sawadogo · A. Ouattara · BE Savadogo-Komboigo Département de gynécologie et d'obstétrique du Centre hospitalier universitaire Yalgado Ouédraogo, 03 BP 7022

Ouagadougou 03, Burkina Faso

e-mail : zamanehyacinthe@gmail.com

H. Zamané · S. Kiemtoré · P.D. Kain · Y.A. Sawadogo

A. Ouattara · I. Ouédraogo · C.M.R. Ouédraogo

Unité de formation et de recherche en sciences de la santé,

Université Ouaga 1 Pr Joseph KI ZERBO, 01 BP 7021

Ouagadougou 01, Burkina Faso

\author{
A. Zongo \\ Ministère de la Santé, Burkina Faso \\ I. Ouédraogo \\ Centre hospitalier universitaire régional de Ouahigouya, \\ Burkina Faso
}

C.M.R. Ouédraogo

Hôpital de District de Bogodogo, Burkina Faso présentation de siège) de la classification de Robson ont toutes bénéficié d'une césarienne et ont contribué au taux global de césarienne pour respectivement $30 \%$ et $8,6 \%$. La contribution relative cumulée au taux global de césarienne des groupes 1, 2, 3 et 4 (femmes à bas risque de césarienne) était de $31,3 \%$. L'amélioration de l'évaluation anténatale du pronostic de l'accouchement, notamment en cas de cicatrice utérine ou de présentation de siège, l'amélioration de la qualité de la surveillance du travail d'accouchement et la lutte contre la prématurité contribueront à maitriser le taux de césarienne à l'hôpital de district de Bogodogo.

Mots clés Césarienne $\cdot$ Abus · Risque obstétrical · Classification de Robson · Hôpital · Bogodogo ·

Ouagadougou $\cdot$ Burkina Faso

Abstract The control of the caesarean rate is nowadays an important concern for the obstetric world, the priority being to make every effort to practice a caesarean in all the women who need it only instead of reaching a specific rate. The purpose of the present study was to apply the Robson classification to the evaluation of the practice of caesarean section at the maternity of the Bogodogo District Hospital. It turned to be an analytical cross-sectional study which was carried out from January 1st, 2013 till December $31^{\text {st }}, 2015$. The information sources used included the computer base of caesarean sections, the delivery records, the operating room records, the delivery hall and the monthly activity reports. The overall hospital frequency of caesarean section was $33.3 \%$. The rate of caesarean section expected during the same period according to the C-Model was $9.7 \%$. Patients in groups 5 (with a scar uterus) and 6 (nulliparous with siege presentation) of the Robson classification had all a caesarean section and contributed to the overall rate of caesarean for 30 and $8.6 \%$ respectively. Low-risk women (groups 1, 2 , 3 and 4 ) had a relative contribution of $31.3 \%$ to the overall 
rate of caesarean section. Improvement of the antenatal assessment of the prognosis of childbirth, particularly in the case of uterine scar or siege presentation, improvement of the quality of the supervision of the delivery work and the fight against prematurity will help to control the rate of caesarean section at the Bogodogo District Hospital.

Keywords Practice - Caesarian - Robson classification · Hospital $\cdot$ Bogodogo $\cdot$ Ouagadougou $\cdot$ Burkina Faso

\section{Introduction}

La césarienne est l'un des actes les plus pratiqués en obstétrique [24]. Sa pratique est relativement facile ce qui peut entraîner un risque d'abus. Du fait des risques inhérents à la césarienne $[6,8,11,18]$, le contrôle de son taux constitue une préoccupation importante dans le domaine obstétrical. La priorité est, de nos jours, de tout mettre en œuvre pour pratiquer une césarienne chez toutes les femmes qui en ont besoin, plutôt que d'atteindre un taux spécifique comme c'était la cas antérieurement [20]. Pour l'heure, en l'absence de système de classification mondialement reconnu pour la césarienne qui permettrait de réaliser des comparaisons pertinentes et significatives des taux de césarienne entre différents établissements de soins, villes ou régions, l'Organisation mondiale de la santé (OMS) propose d'adopter la classification en 10 groupes, dite de Robson [16,20]. Cette classification a la particularité d'analyser les taux de césarienne par sous-groupes présentant différents niveaux de risque, de savoir quelle est la contribution de chacun de ces groupes au taux global de césarienne afin d'identifier ceux sur lesquels faire porter les efforts et de proposer des mesures concrètes pour éviter les césariennes non nécessaires $[16,20,23]$. Cette stratégie est définie dans l'optique de produire des taux ajustés qui tiennent compte de la réalité des maternités [2,29]. Au Burkina Faso, l'accessibilité financière de la césarienne est en amélioration du fait essentiellement de la politique de subvention, puis de gratuité des soins obstétricaux et néonatals d'urgence. Son taux y est alors en augmentation progressive en milieu hospitalier [12] alors que le plateau technique pour sa réalisation est encore loin de la norme. L'hôpital de district de Bogodogo en est un reflet [21]. La présente étude avait pour objectif d'appliquer la classification de Robson pour évaluer la pratique de la césarienne à la maternité de cet hôpital de 2013 à 2015.

\section{Patientes et méthodes}

Il s'est agi d'une étude transversale à collecte rétrospective qui a concerné une période de trois ans, du $1^{\mathrm{er}}$ janvier 2013 au 31 décembre 2015. Les patientes ayant accouché à la maternité de l'hôpital de district de Bogodogo pendant cette période ont constitué la population de l'étude.

La base informatique des dossiers de césarienne, les dossiers d'accouchement, les registres du bloc opératoire, de la salle d'accouchement et les rapports mensuels d'activités étaient les sources d'information utilisées. Les données ont été collectées par une enquêtrice, étudiante en année de thèse de médecine, aidée de l'informaticienne en charge de la gestion des dossiers informatisés de l'hôpital. Cette enquêtrice a reçu au préalable une formation sur la classification de Robson. Pour classer les femmes dans les 10 groupes, un script a été fait à partir des variables essentielles, à savoir la parité, les antécédents de césarienne, le nombre de fœtus, le type de début du travail, la présentation du fœetus, l'âge gestationnel et la voie d'accouchement. La classification de Robson a permis de distinguer les femmes selon différents niveaux de risque obstétrical, les 4 premiers groupes ayant été considérés comme étant composés de femmes à bas risque de césarienne et les 6 autres de femmes à risque élevé. Le taux attendu de césarienne a été déterminé à l'aide du modèle prédictif (C-model) de Souza [28]. Ce taux est la moyenne des probabilités individuelles des femmes à bénéficier d'une césarienne. Les probabilités ont été déterminées à partir du modèle mathématique logit qui s'écrit : Logit $=\beta_{0}+\beta_{1} X_{1}+\beta_{2} X_{2}+\ldots+\beta_{n} X_{n}$ (avec $\beta_{0}=$ constante, $X=$ variable du C-Model). Les variables du modèle étaient les caractéristiques obstétricales des femmes prisent en compte dans la classification de Robson : âge, morbidité maternelle sévère (la défaillance viscérale, l'admission en soins intensifs) et présence de complications dont le placenta prævia, l'hématome rétro-placentaire, l'hypertension chronique, la pré-éclampsie/éclampsie, la pathologie rénale et le VIH.

\section{Résultats}

\section{Fréquence de la césarienne}

Sur un total de 9068 accouchements, 3020 cas de césariennes ont été enrégistrés, soit une fréquence globale hospitalière observée de $33,3 \%$. La fréquence des césariennes a augmenté de 2013 à 2014, puis a connu une dimunition significative en 2015 par rapport à $2013(p<0,0005)$. L'évolution de ce taux de césarienne attendu durant la période d'étude (la moyenne des probabilités individuelles générées par le C-Model) était de 9,7\% (tableau 1).

\section{Classification de Robson}

Quatre-vingt-dix-sept femmes n'ont pas été classées du fait de données manquantes (parité non précisée). Les patientes du groupe 5 (multipares avec antécédent de césarienne, singleton céphalique, $\geq 37 \mathrm{SA}$ ) ont accouché par voie haute dans 
Tableau 1 Fréquence de la césarienne de 2013 à 2015 à la maternité du district sanitaire de Bogodogo/ Frequency of caesarean section from 2013 to 2015 at the maternity ward of the Bogodogo District Hospital

\begin{tabular}{|llll|}
\hline Année & Nombre d'accouchements & Nombre de césariennes & $\begin{array}{l}\text { Fréquence relative } \\
\text { des césariennes \% }\end{array}$ \\
\hline 2013 & 2880 & 936 & 32,5 \\
2014 & 3018 & 1114 & 36,9 \\
2015 & 3170 & 970 & 30,6 \\
Total & $\mathbf{9 0 6 8}$ & $\mathbf{3 0 2 0}$ & $\mathbf{3 3 , 3}$ \\
\hline
\end{tabular}

$100 \%$ des cas. La contribution relative du groupe au taux global de césarienne était de $30 \%$ (tableau 2).

Les femmes à bas risque (groupes $1,2,3,4$ ) avaient une contribution relative cumulée de $31,3 \%$ au taux de césarienne.

\section{Indications de césarienne}

Les indications de césarienne étaient dominées par la cicatrice utérine. L'asphysie fœtale et la disproportion fœtopelvienne étaient repsectivement les deuxième et troisième plus fréquentes indications relevées (tableau 3).

\section{Discussion}

La classification de Robson ne tenant pas compte des pathologies préexistantes, des indications et méthodes de déclenchement du travail ainsi que du degré de prématurité, ces facteurs n'ont pas été pris en considération dans l'analyse bien que leur influence sur le taux de césarienne soit notable. Cet état de fait peut constituer une limite dans l'appréciation du taux de pratique de césarienne. La fréquence globale des césariennes était élevée $(33,3 \%)$ pour une fréquence attendue de $9,7 \%$. Cette fréquence a significativement varié d'une année à l'autre. Elle était en augmentation de 2013 à 2014. Par contre en 2015, il a été noté une baisse significative du taux de césarienne. Cette baisse pourrait être en rapport avec la fermeture fréquente et prolongée du bloc opératoire, suite aux troubles socio-politiques qu'a connu le pays en 2015. La fréquence élevée de césariennes dans notre série pourrait s'expliquer en grande partie par le fait que l'hôpital du district de Bogodogo demeure un important centre de référence des urgences obstétricales en provenance de multiples centres périphériques. Toutefois, cet hopital ne repond pas aux normes recommandées pour une salle de naissance du fait d'un sous-équipement marqué. Plusieurs césariennes y sont pratiquées sans une raison médicale évidente et ceci par crainte de l'issue présumée défavorable de l'accouchement par la voie basse. Des études antérieures ménées par Kouanda et al [15] ainsi que par Kaboré et al [14] ont relevé la part importante de césariennes non médicalement justifiées à l'échelle du pays.
Aussi l'amélioration de l'accès de la population à la césarienne par le renforcement des capacités des structures en soins obstétricaux et néonatals d'urgence (SONU) et la politique de subvention de ces soins sont en faveur de l'augmentation des taux de césarienne aussi bien en population qu'en institution dans la plupart des pays d'Afrique sub-saharienne [5,9,22,27,31]. En effet, Schantz et al [25] relevaient un taux élevé de césarienne dans une étude multicentrique menée au Mali (31\%) et au Bénin (43,9 \%) où une politique d'exemption de paiement de frais de soins obstétricaux et néonatals d'urgence était mis en œuvre.

De l'analyse selon la classification de Robson, le premier contributeur au taux global de césarienne était le groupe 5 (multipares avec antécédents de césarienne, singleton en céphalique, âge gestationnel $\geq 37 \mathrm{SA}$ ) avec $30 \%$. Aussi, $100 \%$ des patientes de ce groupe avaient accouché par césarienne. La forte contribution de ce groupe au taux global de césarienne dans le contexte africain a été relevée par Schantz et al [25] au Mali et au Bénin (respectivement 25,3\% et 29,6 \%) ainsi que par Vogel et al [30] au Kenya, au Nigeria, en Ouganda, en République démocratique du Congo et au Niger. Cela pose la problématique de la conduite du travail d'accouchement sur utérus cicatriciel dans notre contexte ainsi que dans les autres pays à ressources limitées. En effet, un utérus cicatriciel a été retrouvé dans $25,9 \%$ des cas de césarienne. Pourtant, la césarienne itérative ne devrait s'imposer absolument que si la cause de la première césarienne demeure permanente. Il existe en fait peu de situations où la seule cicatrice utérine contre-indique de façon formelle une voie basse qui aurait été acceptée en l'absence de cicatrice. La seule contre-indication formelle réelle demeure l'antécédent de cicatrice corporéale qui s'accompagne d'un risque majeur de rupture utérine [7]. Toutefois, il est à noter que le risque de rupture utérine augmente en cas d'utérus multi-cicatriciel ou de délai court. La connaissance de la technique opératoire et des suites opératoires est indispensable pour le pronostic des accouchements ultérieurs. Dans notre contexte, ces informations médicales permettant l'appréciation de la cicatrice utérine (compte rendu opératoire, suites opératoires) sont peu disponibles surtout en situation d'urgence où les décisions de la voie d'accouchement sont le plus souvent prises. De plus, on constate un manque de 
Tableau 2 Distribution du taux de césarienne selon la classification de Robson et la contribution au taux de césarienne / Distribution of caesarean rate according to Robson classification and contribution to the rate of caesarean section.

\begin{tabular}{|c|c|c|c|c|c|}
\hline Groupes de Robson & $\begin{array}{l}\text { Nbr } \\
\text { de césarienne / } \\
\text { Nbr de femme }\end{array}$ & $\begin{array}{l}\text { Taille relative } \\
\text { de la classe }(\%)\end{array}$ & $\begin{array}{l}\text { Taux } \\
\text { de césarienne } \\
(\%)\end{array}$ & $\begin{array}{l}\text { Contribution } \\
\text { relative }(\%)\end{array}$ & $\begin{array}{l}\text { Contribution } \\
\text { absolue (\%) }\end{array}$ \\
\hline $\begin{array}{l}\text { Groupe } 1 \text { : nullipare, grossesse } \\
\text { unique, présentation céphalique, âge } \\
\text { gestationnel } \geq 37 \text { semaines, travail } \\
\text { spontané }\end{array}$ & $247 / 2692$ & 30,0 & 9,1 & 8,2 & 2,7 \\
\hline $\begin{array}{l}\text { Groupe } 2 \text { : nullipare, grossesse } \\
\text { unique, présentation céphalique, âge } \\
\text { gestationnel } \geq 37 \text { semaines, } \\
\text { déclenchement du travail } \\
\text { ou césarienne avant travail }\end{array}$ & $220 / 1004$ & 11,2 & 21,9 & 7,3 & 2,4 \\
\hline $\begin{array}{l}\text { Groupe } 3 \text { : multipares, sans utérus } \\
\text { cicatriciel, grossesse unique, } \\
\text { présentation céphalique, âge } \\
\text { gestationnel } \geq 37 \text { semaines, travail } \\
\text { spontané }\end{array}$ & $256 / 1291$ & 14,4 & 19,8 & 8,5 & 2,8 \\
\hline $\begin{array}{l}\text { Groupe } 4 \text { : multipares, sans utérus } \\
\text { cicatriciel, grossesse unique, } \\
\text { présentation céphalique, âge } \\
\text { gestationnel } \geq 37 \text { semaines, } \\
\text { déclenchement du travail } \\
\text { ou césarienne avant travail }\end{array}$ & $220 / 1117$ & 12,4 & 19,7 & 7,3 & 2,4 \\
\hline $\begin{array}{l}\text { Groupe } 5 \text { : toutes les multipares } \\
\text { avec au moins une cicatrice utérine, } \\
\text { grossesse unique, présentation } \\
\text { céphalique, âge gestationnel } \geq \\
37 \text { semaines, travail spontané }\end{array}$ & $906 / 906$ & 10,1 & 100,0 & 30,0 & 10,1 \\
\hline $\begin{array}{l}\text { Groupe } 6 \text { : toutes les nullipares, } \\
\text { grossesse unique, présentation } \\
\text { du siège }\end{array}$ & $260 / 260$ & 2,9 & 100,0 & 8,6 & 2,9 \\
\hline $\begin{array}{l}\text { Groupe } 7 \text { : toutes les multipares, } \\
\text { grossesse unique, présentation } \\
\text { du siège, utérus cicatriciel inclus }\end{array}$ & $101 / 101$ & 1,1 & 100,0 & 3,3 & 1,1 \\
\hline $\begin{array}{l}\text { Groupe } 8: \text { toutes les grossesses } \\
\text { multiples, utérus cicatriciel inclus }\end{array}$ & $220 / 806$ & 8,9 & 27,2 & 7,3 & 2,4 \\
\hline $\begin{array}{l}\text { Groupe } 9 \text { : toutes les grossesses } \\
\text { uniques avec présentation transverse } \\
\text { ou oblique, utérus cicatriciel inclus }\end{array}$ & $64 / 64$ & 0,7 & 100,0 & 2,1 & 0,7 \\
\hline $\begin{array}{l}\text { Groupe } 10 \text { : toutes les grossesses } \\
\text { uniques avec présentation céphlique, } \\
\text { âge gestationnel }<37 \text { semaines, } \\
\text { utérus cicatriciel inclus }\end{array}$ & $526 / 730$ & 8,1 & 72,0 & 17,4 & 5,9 \\
\hline Total & $3020 / 8971$ & & & 100,0 & 33,3 \\
\hline
\end{tabular}

moyens de surveillance plus adaptés lors de l'épreuve utérine, notamment la tocographie interne comme également relevé par Schantz et al [25]. La tentative d'accouchement par voie basse sur utérus cicatriciel demeure de nos jours l'une des préoccupations majeures de l'obstétricien dans les pays en développement. Toutefois il convient de rappeler qu'avec une bonne surveillance prénatale et per partum, l'épreuve utérine n'aggrave pas le pronostic maternel et 
Tableau 3 Répartition des cas de césarienne selon la principale indication $(n=3020) /$ Distribution of caesarean section cases according to the main indication $(n=3020)$.

\begin{tabular}{|lll|}
\hline Indications & N & \% \\
\hline Utérus cicatriciel & 782 & 25,9 \\
Asphyxie fœtale & 711 & 23,5 \\
Disproportion foto-pelvienne & 663 & 21,9 \\
Pré-éclampsie/éclampsie & 293 & 9,7 \\
Siège & 255 & 8,4 \\
Pré-rupture utérine & 51 & 1,7 \\
Grossesse multiple & 39 & 1,3 \\
Rupture prématurée & 39 & 1,3 \\
des membranes & & \\
Présentation dystocique & 33 & 1,1 \\
Procidence du cordon & 32 & 1,0 \\
Hydramnios/oligoamnios & 31 & 1,0 \\
Dépassement de terme & 29 & 0,9 \\
Placenta prævia & 21 & 0,7 \\
Dilatation stationnaire & 16 & 0,5 \\
Hématome retro placentaire & 13 & 0,4 \\
Dystocie dynamique & 5 & 0,1 \\
irréductible & & 0,2 \\
Autres & 7 & $\mathbf{1 0 0}$ \\
Total & $\mathbf{3 0 2 0}$ & \\
\hline
\end{tabular}

périnatal [13]. Pour une bonne pratique obstétricale, il est important que dans la mesure du possible, l'accouchement par voie basse soit priviligié pour réduire les utérus multicicatriciels surtout dans une société pronataliste où l'indice synthétique de fécondité reste encore élevé comme celui des auteurs (5,4 en 2015) [17].

Les femmes avec singletons prématurés en présentation céphalique utérus cicatriciel inclus (groupe 10) sont également de grandes contributrices $(17,4 \%), 72 \%$ des femmes de ce groupe ayant accouché par césarienne. Ce taux s'expliquerait probablement par une part plus importante de prématurité induite pour des pathologies maternelles (HTA non contrôlée au cours de la grossesse, diabète non stabilisé), indiquant en elles-mêmes la réalisation d'une césarienne. À ceci s'ajoute la fréquence dans notre contexte des infections vaginales souvent incriminées dans les accouchements prématurés avec une limitation des moyens de maturation du col en toute sécurité.

Les nullipares avec singleton et présentation de siège (groupe 6) ont contribué au taux global de césarienne pour $8,6 \%$. La pratique de la césarienne était systématique dans ce groupe $(100 \%)$. Ce type de présentation a été en cause dans $8,4 \%$ de nos cas de césarienne. La présentation du siège a été moins impliquée dans les séries de Cissé et al ainsi que de Sépou et al respectivement au Sénégal et en République centrafricaine $[4,26]$. La voie d'accouchement dans la présentation du siège reste encore un sujet à controverse. En effet, si certains auteurs plaident en faveur d'une césarienne systématique [10], d'autres à l'inverse, semblent être en faveur d'une possible acceptation de la voie basse dans le cadre d'une sélection rigoureuse des patientes et ceci sans augmentation réelle de la morbidité et de la mortalité néonatale, mais avec un gain maternel considérable $[1,3,19]$. La présentation du siège ne saurait donc être qualifiée de dystocique a priori, mais plutôt de potentiellement dystocique. Pour autoriser la voie basse dans les présentations du siège, il est important entre autres de réaliser une évaluation préalable du bassin, de préférence par la radiopelvimétrie au troisème trimestre de la grossesse. Dans le contexte local, cet examen est peu disponible et les parturientes sont en général reçues en urgence, sans avoir bénéficié au préalable de cette exploration. Cela peut expliquer le recours fréquent de beaucoup de praticiens à la césarienne de sécurité chez les nullipares avec foetus en siège.

Les femmes à bas risque (groupes 1, 2, 3,4) ont contribué à environ un tiers du taux global de césarienne $(31,3 \%)$. Ceci pourrait s'expliquer par un nombre non négligeable de ces femmes au sein de la population générale. Parmi ces groupes de femmes à bas risque, le taux le plus élevé de césarienne était de 21,9\% supérieur au taux prédit par le C-Model, ce qui montre qu'il y a eu des césariennes évitables. L'asphyxie fotale était la deuxième plus fréquente indication $(23,5 \%)$ après la cicatrice utérine $(25,9 \%)$. C'est le lieu de rappeler l'intérêt de l'utilisation des méthodes alternatives à la césarienne dont l'extraction instrumentale, en vue de réduire le délai d'expulsion en cas de suspicion de souffrance fœtale aiguë sur un travail d'accouchement avancé. Aussi, le diagnostic de l'asphyxie fotale était fait sur la base de l'auscultation intermittente à l'aide du stéthoscope de Pinard ou parfois de la seule appréciation de la coloration du liquide amniotique. Cela peut conduire à un risque d'erreur diagnostique par excès et par conséquent à des césariennes évitables. Dans notre série, le score d'Apgar à la cinquième minute chez les nouveau-nés issus de la césarienne était supérieur ou égal à 7 dans la très grande majorité des cas $(95,6 \%)$, ce qui permet une remise en cause du diagnostic de certains cas d'asphyxie foetale.

L'étude a favorisé le renforcement du dispositif de surveillance du travail et de conduite de l'accouchement à la maternité du district de Bogodogo, par l'acquisition d'une part, de cardiotocographes, et d'autre part, de ventouses et la formation du personnel à leur utilisation. Aussi, la classification des parturientes selon les groupes de Robson est désormais systématique dès leur admission dans la maternité de l'étude, même si l'évaluation du taux de césarienne n'y est pas encore faite en routine. Toutefois, l'impact réel de l'intervention reste à apprécier par la comparaison de l'évolution du taux de césarienne à l'hôpital de district de Bogodogo à celle d'autres maternités de référence n'ayant pas conduit une telle évaluation. 


\section{Conclusion}

La césarienne occupe une place trop importante dans les activités du service de maternité de l'hôpital du district de Bogodogo. L'amélioration de l'évaluation anténatale du pronostic de l'accouchement, notamment en cas de cicatrice utérine ou de présentation de siège, l'amélioration de la qualité de la surveillance du travail d'accouchement et la lutte contre la prématurité, contribueront à y maîtriser son taux.

Liens d'intérêts Les auteurs déclarent ne pas avoir de liens d'intérêts.

\section{Références}

1. Alarab M, Regan C, O'connell MP, et al (2004) Singleton vaginal breech delivery at term: still a safe option. Obstet Gynecol 103:407-12

2. Betrán AP, Merialdi M, Lauer JA, et al (2007) Rates of caesarean section: analysis of global, regional and national estimates. Paediatr Perinat Epidemiol 21:98-113

3. Carayol M, Alexander S, Goffinet F, et al (2004) Mode d'accouchement des femmes avec une présentation du siège à terme dans l'étude PREMODA. J Gynecol Obstet Biol Reprod (Paris) 33: 7-44

4. Cissé C, Faye E, De Bernis L, et al (2001) Césarienne au Sénégal : couverture des besoins et qualité des services. Cahier Santé 8:369-77

5. Cissé CT, Ngom PM, Guissé A, et al (2004) Réflexions sur l'évolution des taux de césarienne en milieu africain : exemple du CHU de Dakar entre 1992 et 2001. Gynecol Obstet Fertil 32:210-7

6. Curran EA, O'Neill SM, Cryan JF, et al (2015) Research review: Birth by caesarean section and development of autism spectrum disorder and attention-deficit/hyperactivity disorder: a systematic review and meta-analysis. J Child Psychol Psychiatry 56:500-8. doi: 10.1111/jcpp.12351. Epub 2014 Oct 27.

7. D'Ercole C, Bretelle F, Piéchon L, et al (2000) La césarienne at-elle une indication en cas d'utérus cicatriciel ? J Gynecol Obstet Biol Reprod (Paris) 29:S51-S67

8. Deneux-Tharaux C, Carmona E, Bouvier-Colle MH, Bréart G (2006) Postpartum maternal mortality and cesarean delivery. Obstet Gynecol 108(3 Pt 1):541-8

9. El-Khoury M, Gandaho T, Arur A, et al (2011) Improving Access to Life-saving Maternal Health Services: The Effects of Removing User Fees for Caesareans in Mali. MD: Health Systems 20/20, Abt Associates Inc. $105 \mathrm{p}$

10. Hannah ME, Hannah WJ, Hewson SA, et al (2000) Planned caesarean section versus planned vaginal birth for breech presentation at term: a randomised multicentre trial. Term Breech Trial Collaborative Group. Lancet 356:1375-83

11. Hyde MJ, Modi N (2012) The long-term effects of birth by caesarean section: the case for a randomised controlled trial. Early Hum Dev 88:943-9. doi: 10.1016/j.earlhumdev.2012.09.006. Epub 2012 Oct 1.

12. Institut de Recherche en Sciences de la Santé (IRSS) (2010) Evaluation des Besoins en Soins Obstétricaux et Néonataux d'Urgence, couplée à la cartographie de l'offre de soins en Santé de la Reproduction au Burkina Faso. Ministère de la recherche scientifique et de l'innovation. Burkina Faso. $123 \mathrm{p}$

13. Kaboré C, Chaillet N, Kouanda S, et al (2015) Maternal and perinatal outcomes associated with a trial of labour after previous caesarean section in sub-Saharan countries. BJOG 123:21472155. doi: 10.1111/1471-0528.13615. Epub 2015 Sep 16

14. Kaboré C, Ridde V, Kouanda S, et al (2016) Determinants of nonmedically indicated cesarean deliveries in Burkina Faso. Int J Obstet Gynaecol 135:S58-S63. doi: 10.1016/j.ijgo.2016.08.019.

15. Kouanda S, Coulibaly A, Ouedraogo A, et al (2014) Audit of cesarean delivery in Burkina Faso. Int J Gynecol Obstet 125:214-8. doi: 10.1016/j.ijgo.2013.11.010. Epub 2014 Feb 15

16. Lamy C, Alexander S (2015) L'Organisation mondiale de la santé recommande un enregistrement des données sur la césarienne adapté pour utiliser la classification de Robson en routine. J Gynecol Obstet Biol Reprod (Paris) 44:587-90. doi: 10.1016/ j.jgyn.2015.06.019. Epub 2015 Jun 30

17. Ministère de santé, Burkina Faso (2017) Profil sanitaire complet du Burkina Faso. Module 1 Situation socio-sanitaire du Burkina Faso et mise en œuvre des ODD. $49 \mathrm{p}$

18. Moraitis AA, Oliver-Williams C, Wood AM, et al (2015) previous cesarean delivery and the risk of unexplained stillbirth: retrospective study and meta-analysis. BJOG 122:1467-74. doi: 10.1111/1471-0528.13461. Epub 2015 May 29

19. Mukuku O, Kimbala J, Kizonde J (2014) Accouchement du siège par voie basse: étude de la morbi-mortalité maternelle et néonatale. Pan Afr Med J 17:27. doi: 10.11604/pamj.2014.17.27.2037. eCollection 2014

20. OMS (2015) Déclaration de l'OMS sur les taux de césarienne. $8 \mathrm{p}$

21. Ouédraogo CM, Ouédraogo A, Ouattara A, Lankoandé J (2015) La pratique de la césarienne dans un hôpital de district à Ouagadougou : Aspects, épidémiologiques, cliniques et pronostiques à propos de 3381 cas. Méd Santé Trop 25:194-9

22. Ravit M, Audibert M, Ridde V, et al (2018) Removing user fees to improve access to caesarean delivery: a quasi-experimental evaluation in western Africa. BMJ Glob Health 3:e000558. doi: 10.1136/bmjgh-2017-000558. eCollection 2018

23. Robson M (2015) The Ten Group Classification System (TGCS) a common starting point for more detailed analysis. BJOG 122 (5):701. doi: 10.1111/1471-0528.13267. Epub 2015 Jan 20

24. Rozenberg P (2004) L'élévation du taux de césariennes : un progrès nécessaire de l'obstétrique moderne. J Gynecol Obstet Biol Reprod 33:279-89.

25. Schantz C, Ravit M, Traoré AB, et al (2018) Why are caesarean section rates so high in facilities in Mali and Benin? Sex Reprod Healthe 16:10-4. doi: 10.1016/j.srhc.2018.01.001. Epub 2018 Jan 10

26. Sepou A, Yanza M, Nguembi E, et al (2000) Etude de 299 cas de césariennes pratiquées à l'Hôpital Communautaire de Bangui (Centrafrique). Méd Afr Noire 47:14-18

27. Somé DA, Ouattara S, Barro D, et al (2010) Audit des césariennes en milieu africain. Revue Médicale Rwandaise 68:32-7

28. Souza JP, Betran AP, Dumont A, et al (2016) A global reference for caesarean section rates (C-Model): a multicountry cross-sectional study. BJOG 123:427-36. doi: 10.1111/1471-0528.13509. Epub 2015 Aug 10.

29. Stanton C, Ronsmans C; Baltimore Group on Cesarean (2008) Recommendations for routine reporting on indications for cesarean deliveries in developing countries. Birth 35:204-13. doi: 10.1111/j.1523-536X.2008.00241.x.

30. Vogel JP, Betrán AP, Vindevoghel N, et al (2015) Use of the Robson classification to assess caesarean section trends in 21 countries: a secondary analysis of two WHO multicountry surveys. Lancet Glob Health 3:e260-70. doi: 10.1016/S2214-109X(15)70094-X. Epub 2015 Apr 9

31. Wanyonyi S, Sequeira E, Obura T (2006) Caesarian section rates and perinatal outcome at the Aga Khan University Hospital, Nairobi. East Afr Med J 83:651-8 